\title{
Basic Study of 3-D Non-Invasive Measurement of Temperature Distribution using Ultrasound Images during HIFU Heating
}

\author{
Ryosuke Sakakibara*, ${ }^{*}$, Yasuhiro Shindo ${ }^{2}$, Kazuo Kato ${ }^{3}$, Pak Kon $\mathrm{Choi}^{4}$, Akira Takeuchi ${ }^{5}$ \\ ${ }^{1}$ Graduate School of Science and Technology, Meiji University, 214-8571, Japan \\ ${ }^{2}$ Department of Mechanical Engineering, Toyo University, 350-0815, Japan \\ ${ }^{3}$ Department of Mechanical Engineering Informatics, Meiji University, 214-8571, Japan \\ ${ }^{4}$ Department of Physics, Meiji University, 214-8571, Japan
}

${ }^{5}$ Department of Thermotherapy, Luke Hospital, 104-0045, Japan

\begin{tabular}{l} 
A R T I C L E I N F O \\
\hline Article history: \\
Received: 16 September, 2020 \\
Accepted: 01 December, 2020 \\
Online: 16 December, 2020
\end{tabular}

Keywords:

HIFU

Ultrasound

Non-Invasive Temperature

Measurement

\begin{abstract}
A B S T R A C T
High Intensity Focused Ultrasound (HIFU) was widely used for treating tumors noninvasively. In this treatment, ultrasound is focused on the target volume inside the human body to ablate cancerous tissues and Magnetic Resonance Imaging (MRI) is mainly used to grasp the target position and to measure the temperature distributions around the target. However, MRI is very expensive, and a large space is required.

In this paper, we presented a method for measuring the temperature distribution using an ultrasound diagnostic device, which is inexpensive and commonly used in many clinics, and actually showed the results of heating experiments on a human shaped agar phantom. The proposed method for measuring the temperature distribution around the heated target was conducted by performing image processing on two ultrasound images before and after heating. Furthermore, it was confirmed that it was possible to grasp the three-dimensional temperature distribution from the images in multiple layers. The effectiveness of the temperature distribution measurement results by the proposed method was shown by comparing the temperature measurement results with the infrared thermal camera. The error between the results was approximately $1{ }^{\circ} \mathrm{C}$.

It was found that the non-invasive measurement method of the three-dimensional temperature distribution around the target volume using the ultrasound images was useful for effective HIFU treatments.
\end{abstract}

\section{Introduction}

This paper is an extension of a previous work originally presented at the 21 st edition of the International Conference on Electromagnetics in Advanced Applications (ICEAA 2019) [1].

High Intensity Focused Ultrasound (HIFU) devices are used for treating uterine fibroids, liver tumors, brain tumors, etc. as a minimal invasive treatment method $[2,3]$. As shown in Figure 1, this treatment method focuses on ultrasound waves emitted from the outside of the body to a target inside the body and causes

${ }^{*}$ Corresponding Author: Ryosuke Sakakibara, 1-1-1, Higashi-mita, Tama-ku, Kawasaki, Kanagawa, 214-8571,+81-44-934-7907, rsakakibara9@gmail.com ablation necrosis of the tumor by the thermal energy generated at the focal point. This method is considered to be a treatment method that has a much smaller burden on the patient than conventional open surgery. Focused ultrasound treatment is performed at a high temperature of approximately $70{ }^{\circ} \mathrm{C}$ or higher, and therefore there is a risk of cauterizing normal tissue around the tumor. When performing this treatment, a device with MRI's guidance (MRguided focused ultrasound surgery: MRgFUS) is commonly used. However, a large amount of introduction funds and a large space are required. In addition, this method is not considered to be suitable for cases in which there is metal inside the human body or MRI contrast media cannot be used because the inside of the body is monitored using MR images [4-6]. 
Therefore, in order to perform Focused Ultrasound Surgery (FUS) treatment, non-invasive temperature measurement using an ultrasound diagnostic imaging device that is cheaper and more convenient to move than an MRI device has been previously attempted [7-12]. In this study, the ultimate goal is to establish a method for non-invasively measuring three-dimensional temperature distribution in a living body with high resolution in subpixel units [9]. In the temperature measurement method using ultrasound images, it is a prerequisite that the used ultrasound diagnostic imaging system was designed to draw images with constant ultrasound velocity. The velocity of ultrasound waves changes depending on the temperature of the propagating tissue. Therefore, if there is a temperature change inside the tissue, the ultrasound velocity in that part locally changes, and it appears as an extremely small image displacement on the ultrasound image. The temperature could be measured by detecting this slight displacement with image analysis technology $[8,9]$.

In the present paper, first, the algorithm of two-dimensional temperature distribution measurement using ultrasound images is described. Next, the agar phantom was heated in a temperaturecontrolled water tank, the relationship between the temperature increase of the agar and the displacement of the ultrasound image was described. Next, the temperature distribution was measured from the ultrasound image inside the agar phantom when it was heated using the focused ultrasound heating device. The temperature distribution inside the agar phantom during heating was measured using two types of transducers with different focal lengths. In the previous research, we investigated the basic heating characteristics by measuring the temperature distribution using a cylindrical agar phantom [10]. In this study, we measured a wider region of temperature distributions using a human shaped agar phantom in order to confirm that the proposed temperature measurement method is effective in situations closer to clinical treatment.

From the examination of the above experimental results, the usefulness of temperature distribution measurement using an ultrasound diagnostic imaging system was clarified quantitatively.

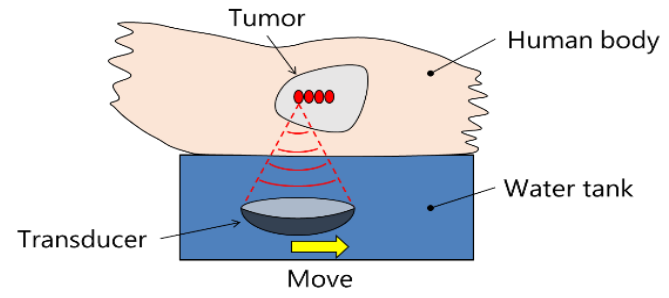

Figure 1: Illustration of Focused Ultrasound Surgery.

\section{Materials and Methods}

\subsection{Principle of Temperature Measurement Using Ultrasound Images}

As reported by Iseki et al., the temperature increases in living tissue can be obtained by solving (1) and (2) $[8,9]$.

$$
\begin{gathered}
\Delta T(x)=K_{\text {tissue }} \frac{\partial(\Delta d)}{\partial x} \\
K_{\text {tissue }}=\frac{1}{\alpha-\beta}
\end{gathered}
$$

In these equations, $\Delta \mathrm{T}$ is the temperature increase inside the tissue and $K_{\text {tissue }}$ is the thermal constant that varies with the tissue. The subscript "tissue" is added to emphasize that it differs depending on the tissue. $\Delta \mathrm{d}$ is the displacement of the ultrasound image due to the temperature increase (the difference between the images before and after heating). The thermal constant $K_{\text {tissue }}$ is expressed by (2). Here, $\alpha$ is the coefficient of thermal expansion of the tissue, and $\beta$ is the coefficient of ultrasound velocity change due to the temperature increase inside the tissue. It is considered that the thermal expansion coefficient $\alpha$ and the ultrasound velocity change coefficient $\beta$ change linearly in a relatively narrow temperature change region such as during focused ultrasound treatment [6].

\subsection{Measurement System of Thermal Constant}

Figure 2 shows the general-purpose ultrasound imaging system Nemio SSA-550A (Toshiba Medical Systems, Japan) with the attached ultrasound probe PLM-805AT (Toshiba Medical Systems, Japan) used in the experiment. The ultrasound probe used in the experiment is a linear probe as shown in Figure 2 (b). The number of elements is 128 , the focus length is $20 \mathrm{~mm}$, and the size of the contact part with the body is $(12 \mathrm{~mm} \times 67 \mathrm{~mm})$. The size of the captured ultrasound image is (560 pixels $\times 450$ pixels), which is a 256-gradation bitmap image. Here, 300 pixels were assigned to a distance of $70 \mathrm{~mm}$ on the captured ultrasound image.

Figure 3 shows an illustration of experimental setup for measuring thermal constant " $K$ " to know the relationship between the temperature increase of the agar used as the heated object and the displacement of the ultrasound images before and after heating $[8,9]$. As shown in Figure 3, a cube agar phantom was fixed inside a water tank where the water temperature was constantly controlled using a thermostat and a stirring pump, and the ultrasound images inside the agar were taken from $20^{\circ} \mathrm{C}$ to $30^{\circ} \mathrm{C}$, because the experiment of temperature distribution measurement is in that temperature range.
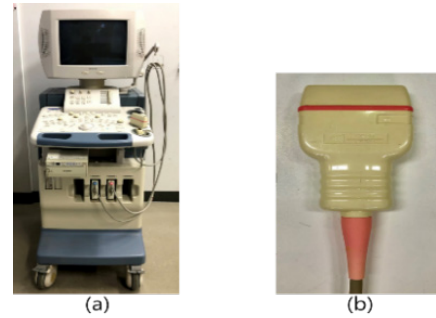

Figure 2: Ultrasound Diagnosis Device (a) Ultrasound Diagnosis Device, (b) Linear Probe.

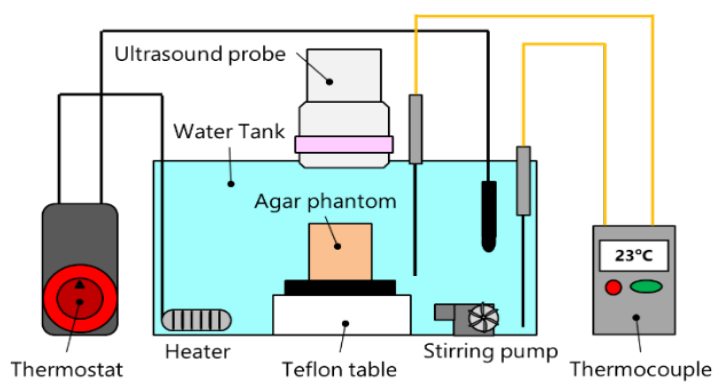

Figure 3: Illustration of Experimental Setup for Measuring Thermal Constant " $K$ "' 


\subsection{Image Analysis Algorithm}

Figure 4 shows the image analysis algorithm proposed by authors for temperature distribution measurement $[8,9]$. This algorithm is divided into 7 steps. First, the ultrasound images of the heated object before and after heating are input. Second, template matching is performed to measure the amount of displacement $\Delta \mathrm{d}$ between the ultrasound images before and after heating. Third, a median filter is applied to reduce the erroneous vector of displacement $\Delta \mathrm{d}$ on the ultrasound image. Fourth, since the displacement $\Delta \mathrm{d}$ distribution detected by template matching is expressed in pixel units, interpolation processing was performed to interpolate $\Delta \mathrm{d}$ distribution. Fifth, a moving average filter is applied to smooth the displacement amount of the distribution map subjected to this interpolation process. Sixth, the temperature increase $\Delta \mathrm{T}$ during heating is calculated from the displacement $\Delta \mathrm{d}$ detected by applying the Sobel filter. Finally, a moving average filter is applied to the temperature increase distribution map to smooth the distribution. For the development of the image analysis program, general-purpose program Microsoft Visual Studio (Microsoft Japan) was used.

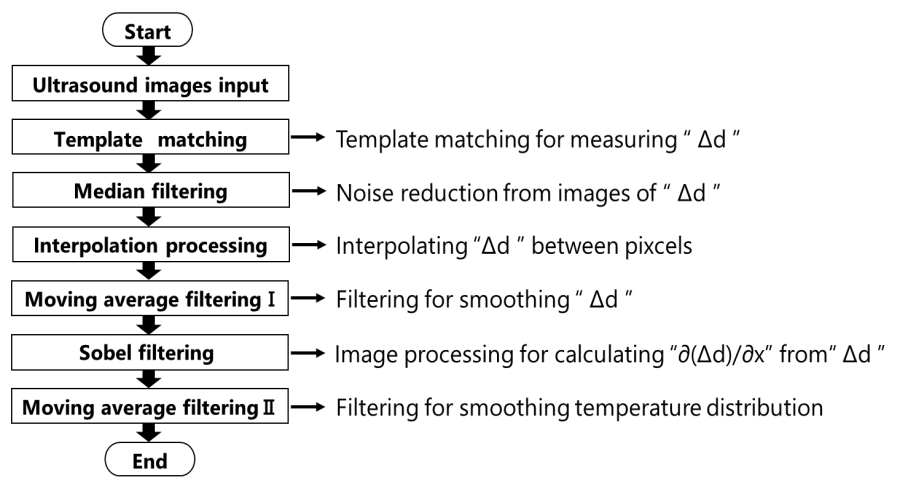

Figure 4: Flow Chart for Measuring Temperature Distributions.

\subsection{Measurement System of Temperature Distributions}

Figure 5 shows the device system for capturing ultrasound images before and after heating. This system mainly consists of two work sliders, an arm and a jig for fixing the ultrasound probe. As shown in Figure 5, the ultrasound probe was supported by the arm and fixed to the slider. Here, the ultrasound probe can be moved three-dimensionally by combining the two sliders. The range of motion of this slider is $(0.0$ to $66.0 \mathrm{~mm})$, and the minimum movable interval is $0.05 \mathrm{~mm}$.

Figure 6 shows an illustration of the focused ultrasound heating experiment. As shown in Figure 6, in order to measure the temperature distribution in the $\mathrm{X}-\mathrm{Z}$ cross section using an infrared thermal camera, the human shaped agar phantom was previously cut at the center position. The transducer was placed underwater to focus on the position $65 \mathrm{~mm}$ from the bottom. Then, the ultrasound probe was moved up and down $\pm 15.0 \mathrm{~mm}$ from the center cross section every $5.0 \mathrm{~mm}$ along the $\mathrm{Z}$ direction, and ultrasound images were taken before and after heating in a total of 7 layers. These operations were performed for heating experiments with short focus and long focus transducers respectively. The heating time was 5 minutes and the heating power was $200 \mathrm{~W}$. In addition, we compared the temperature distribution results by the present method using the ultrasound image and the thermal image taken by the infrared thermal camera in the central section of the human shaped agar phantom.

The focused ultrasound heating device used in the experiments was CZ901 (Mianyang Sonic Electronic, China). Two kinds of transducers were prepared for this device. Figure 7 shows the transducer used in the experiments. The short focus transducer as shown in Figure 7 (a) had an outer diameter of $170 \mathrm{~mm}$, an inner diameter of $98 \mathrm{~mm}$, an outer peripheral surface height of $75 \mathrm{~mm}$, an inner peripheral surface height of $53 \mathrm{~mm}$, and a focal length of $120 \mathrm{~mm}$. The outer and inner diameters of the long focus transducer as shown in Figure 7 (b) were almost the same as those of the short focus transducer, however the inner peripheral surface height was $61 \mathrm{~mm}$ and focal length was $200 \mathrm{~mm}$. The frequency of the focused ultrasound was $1 \mathrm{MHz}$. In the heating experiments, a frequency oscillator AWG1025F (Frequency Band: $1 \mu \mathrm{Hz}-$ 25MHz, AS ONE, Japan) and a power amplifier L-400BM-HC (Frequency Band: $700 \mathrm{kHz}-3.5 \mathrm{MHz}$, JSE, Japan) were connected to the transducers to heat the human shaped agar phantom.

Figure 8 shows the human shaped agar phantom used in the experiments. The width of heating area was $160 \mathrm{~mm}$ and the height was $120 \mathrm{~mm}$.

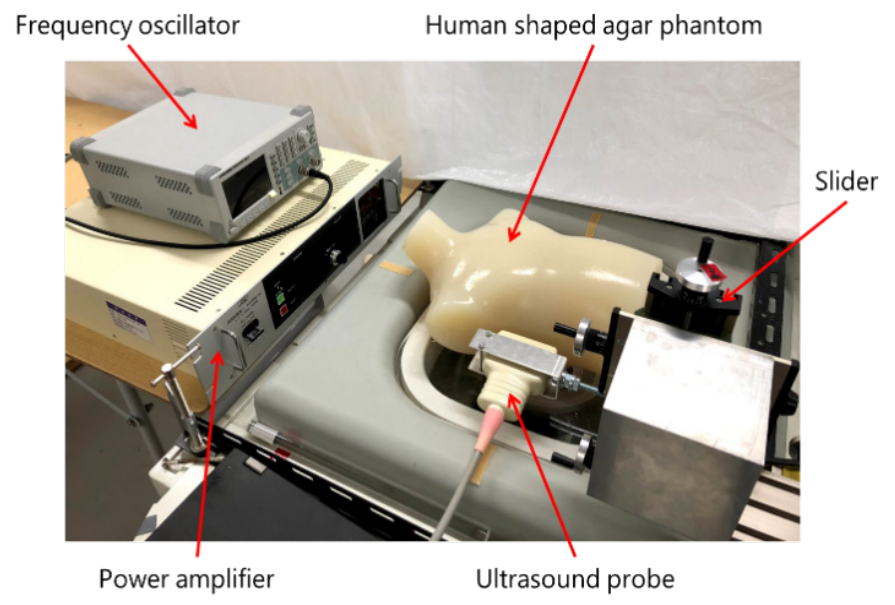

Figure 5: Experimental Setup for Capturing Ultrasound Images.

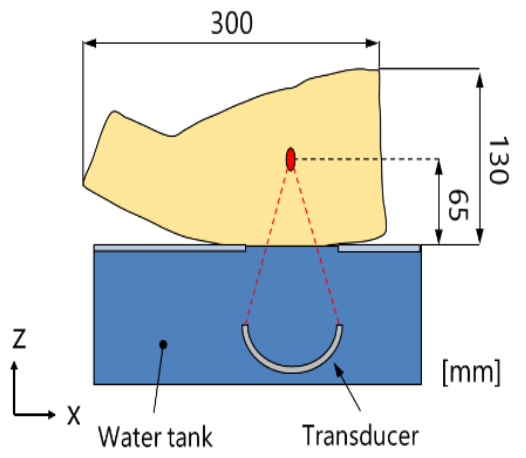

(a)

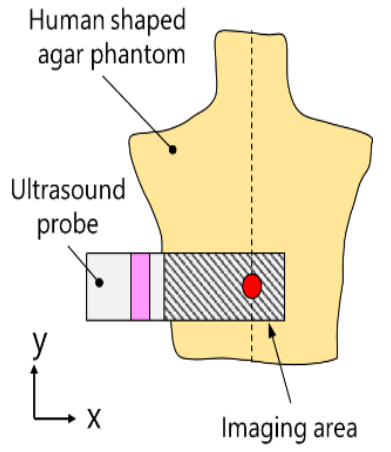

(b)
Figure 6: Illustration of Experimental Setup for Scanning Ultrasound Images. (a) Side View, (b) Top View. 

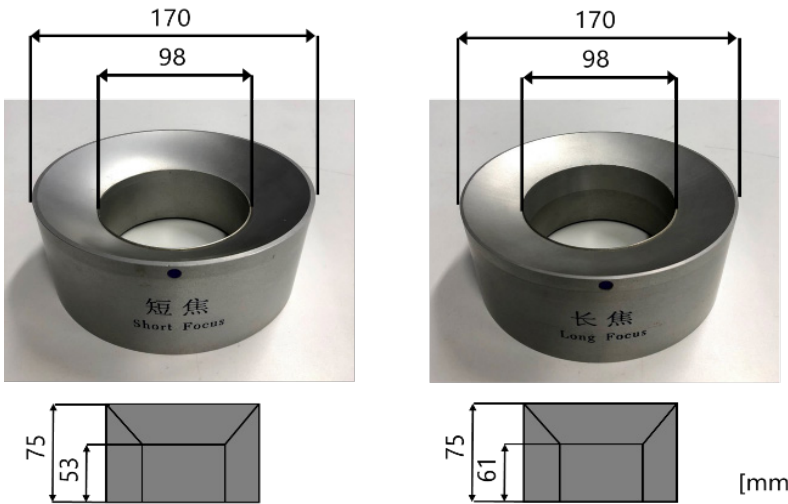

(a)

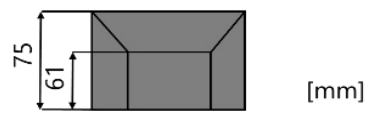

(b)

Figure 7: Transducers Used in the Experiments. (a) Short Focal Length, (b) Long Focal Length.

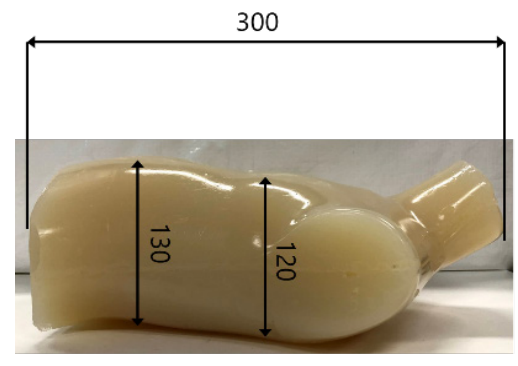

(a)

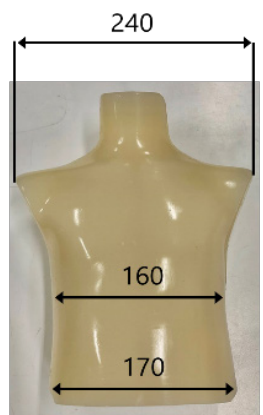

(b)
Figure 8: Human Shaped Agar Phantom.

(a) Side View, (b) Top View.

\section{Results}

\subsection{Thermal Constant of Agar Phantom}

Figure 9 shows the relationship between the temperature change $(\Delta T)$ of the agar phantom and the degree of $(\partial(\Delta d) / \partial x)$ of the ultrasound image when heated as shown in Figure 3 . The template size used in the image analysis as shown in Figure 4 was (13 pixels $\times 13$ pixels). The total number of plots in Figure 9 is 45 . As a result of finding the approximate straight line from these data by the method of least squares, the slope of the graph was 102.92 and the intercept was -0.5262 . The thermal constant of the agar phantom used in this heating experiment was set to $K_{\text {agar }}=102.92$. In addition, the coefficient of determination $\mathrm{R}^{2}$, which is the degree of fitting of the approximate straight line, is 0.9941, which confirms that there is a high correlation.

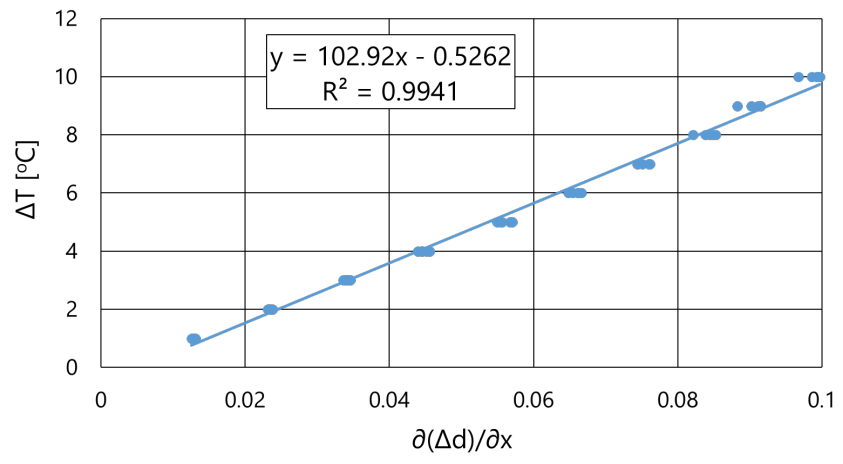

Figure 9: Relationship between $\Delta \mathrm{T}$ and $\partial(\Delta \mathrm{d}) / \partial \mathrm{x}$.

\subsection{2-D Temperature Distributions}

Figure 10 shows the comparison results of the temperature distribution in the central cross section of the human shaped agar phantom when heated with a short focus transducer. In the temperature distribution measurement result from the ultrasound image as shown in Figure 10 (a), the maximum temperature increase value $\Delta \mathrm{T}_{\max }$ was $10.1^{\circ} \mathrm{C}$. On the other hand, it was 10.8 ${ }^{\circ} \mathrm{C}$ from the infrared thermal camera as shown in Figure 10 (b). The measured temperature error by both methods was $0.7^{\circ} \mathrm{C}$, and the error rate was approximately $7 \%$. When comparing the absolute temperatures of both during and after heating, an error naturally occurs. However, it is useful to know the temperature distribution patterns.

Figure 11 shows the temperature in the center section of the agar phantom when the same heating was performed using the long focus transducer. In the temperature distribution measurement result from the ultrasound image as shown in Figure 11 (a), the maximum temperature increase $\Delta \mathrm{T}_{\max }$ was $7.2^{\circ} \mathrm{C}$. On the other hand, it was $5.9^{\circ} \mathrm{C}$ from the image of the infrared thermal camera as shown in Figure. 11 (b). The measured temperature error was $1.3{ }^{\circ} \mathrm{C}$, and the error rate was approximately $18 \%$.

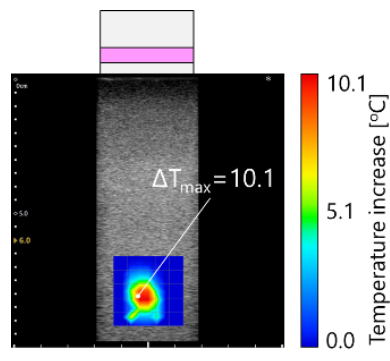

(a)

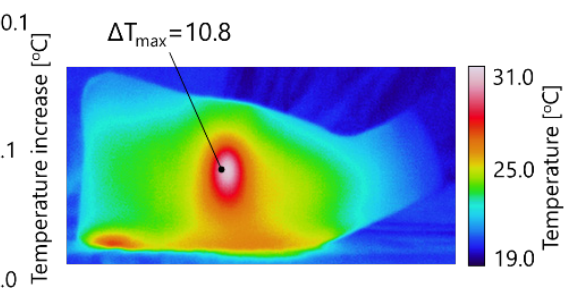

(b)
Figure 10: Comparison between Temperature Distributions inside Human Shaped Agar Phantom (Short Focal Length). (a) with Ultrasound Image, (b) with Thermal Camera

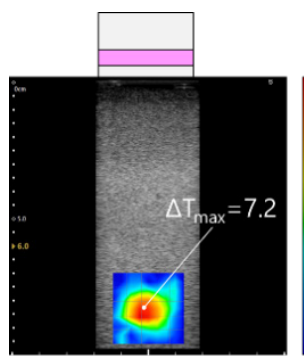

(a)

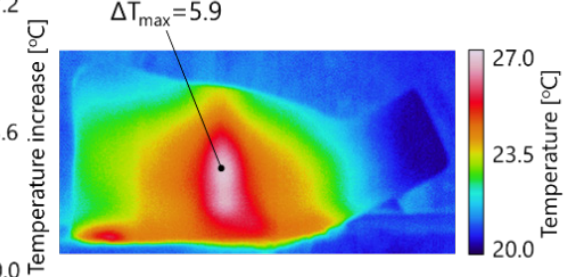

(b)

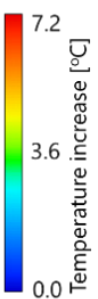

Figure 11: Comparison between Temperature Distributions inside Human Shaped Agar Phantom (Long Focal Length). (a) with Ultrasound Image, (b) with Thermal Camera.

\subsection{3-D Temperature Distributions}

Figure 12 shows the two-dimensional temperature distribution results measured from the ultrasound images of the top and bottom $( \pm 15.0 \mathrm{~mm})$ taken at $5.0 \mathrm{~mm}$ intervals in the center section of the agar phantom. As shown in Figure 12, the two-dimensional temperature distribution results are three-dimensionally displayed by stacking them. Figure 12 (a) shows the case when using the short focus transducer. It can be confirmed that the temperature increase is the highest value in the central cross section and 
decreases with increasing distance from the central cross section, and there is almost no temperature increase above $\pm 10.0 \mathrm{~mm}$. On the other hand, Figure 12 (b) shows the case when using a long focus transducer. Also in this case, it can be seen that the temperature increase is the highest value in the central cross section. However, in the other image layers, unlike the case when the short focus transducer was used, it can be confirmed that the heating region is wide and the temperature increase value at \pm 10.0 $\mathrm{mm}$ is similar to the value at the center cross section.

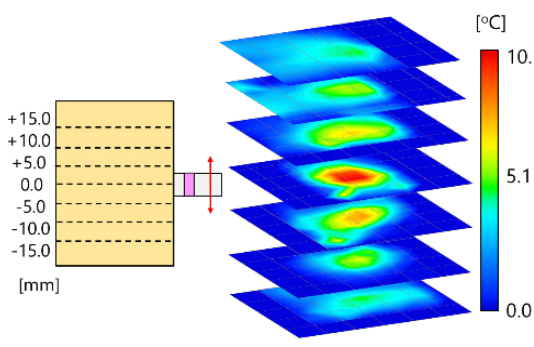

(a)

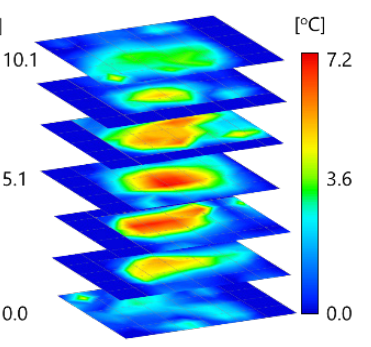

(b)
Figure 12: Temperature Distributions from Ultrasound Images. (a) Short Focal Length, (b) Long Focal Length.

\section{Discussion}

In order to improve the accuracy in the temperature distribution measurement method of applying ultrasound images, we have been working on, (i) taking the images before and after heating at the same position, and (ii) grasping the thermal constant $K_{\text {tissue }}$ of the targeted object. Regarding item (i), as shown in Figure 5, the ultrasound probe could be moved three-dimensionally by using two sliders with a movement accuracy of $\pm 0.05 \mathrm{~mm}$. Regarding item (ii), the value of the agar phantom was actually measured using the self-made device as shown in Figure 3. However, in the results shown in Figure 9, the variation of the measured 45 data was large. It is necessary to control the temperature of the object more accurately by using a higher quality thermostat.

In clinical HIFU treatments, the cauterization temperature of the tumor is mainly $70{ }^{\circ} \mathrm{C}-80{ }^{\circ} \mathrm{C}$. The human shaped agar phantom used in the HIFU experiments was prepared with the component ratio recommended by the Japan Hyperthermia Society. When the maximum temperature near the focal point, where ultrasound waves were focused, was $50{ }^{\circ} \mathrm{C}$ or higher, the agar phantom melted. Therefore, heating experiments of the agar phantom were conducted at $50{ }^{\circ} \mathrm{C}$ or lower. As shown in Figure 12 (a) and (b), heating results using transducers with different focal lengths had different maximum temperatures. Therefore, the normalized temperature $\left(\Delta \mathrm{T}_{\mathrm{N}}\right)$ shown in the following (3) was calculated, and the heating characteristics of both transducers were compared.

$$
\Delta T_{N}=\frac{\Delta T-\Delta T_{\min }}{\Delta T_{\max }-\Delta T_{\min }}
$$

Here, $\Delta T_{\max }$ is the maximum temperature increase value and $\Delta \mathrm{T}_{\min }$ is the minimum temperature increase value in each layer as shown in Figure 12.

Figure 13 shows the calculation results of the normalized temperature $\left(\Delta \mathrm{T}_{\mathrm{N}}\right)$. As shown in Figure 13, when a short focus transducer is used, the temperature increase at a position about \pm $10 \mathrm{~mm}$ away from the focal point is $60 \%$ or less of that focal point. www.astesj.com
That is, when the maximum temperature at the focal point is $80^{\circ} \mathrm{C}$, the temperature at the distance $( \pm 10 \mathrm{~mm})$ is approximately $50^{\circ} \mathrm{C}$. However, when a long focus transducer is used, the temperature at the same distance is approximately $60{ }^{\circ} \mathrm{C}$, and the difference in temperature between them is $10{ }^{\circ} \mathrm{C}$. From this result, careful attention is required at the boundary between the healthy tissue and the tumor tissue during HIFU treatments.

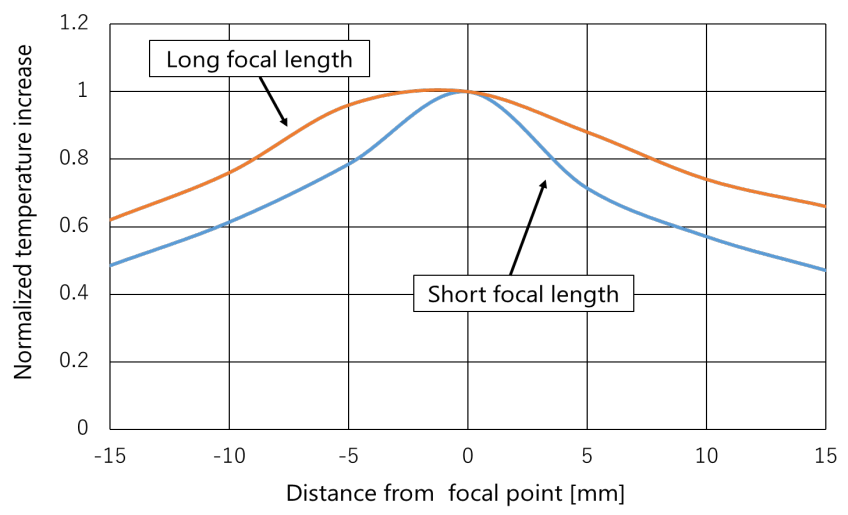

Figure 13: Normalized Temperature Profiles.

\section{Conclusion}

In this paper, we investigated the possibility of non-invasive measurement of the three-dimensional temperature distribution of the human shaped agar phantom during focused ultrasound heating from ultrasound images before and after heating. First, an algorithm of temperature distribution measurement using ultrasound images was presented. Second, the relationship between the temperature increase inside the heated object and the displacement of the ultrasound images before and after heating was described. Third, we measured the temperature distribution inside the agar phantom during HIFU heating using two types of transducers with different focal lengths. In the temperature measurement results, at the central cross section of the agar phantom, the error between the results of the proposed method using ultrasound images and the measurement results of the infrared thermal camera was approximately $1{ }^{\circ} \mathrm{C}$. In addition, in order to discuss the heating characteristics of two types of transducers with different focal lengths, their three-dimensional temperature distributions were compared. Finally, we highlighted the problems in HIFU treatments using transducers with different focal lengths.

In the future, we have a plan to measure the three-dimensional temperature distribution inside heated animals and to discuss the problems of HIFU treatments.

\section{Conflict of Interest}

The authors declare no conflict of interest.

\section{Acknowledgment}

Part of this research is supported by JSPS KAKENHI Grant Number JP18H03553.

\section{References}

[1] R. Sakakibara, K. Kato, P.K. Choi, A. Takeuchi, "Basic study of 3-D Noninvasive measurement of temperature distributions from ultrasound images 
during hifu treatments," in Proceedings of the 2019 21st International Conference on Electromagnetics in Advanced Applications, ICEAA 2019, 2019, doi:10.1109/ICEAA.2019.8879194.

[2] S. Vaezy, R.W. Martin, U. Schmiedl, M. Caps, S. Taylor, K. Beach, S. Carter, P. Kaczkowski, G. Keilman, S. Helton, W. Chandler, P. Mourad, M. Rice, R. Roy, L. Crum, "Liver hemostasis using high-intensity focused ultrasound," Ultrasound in Medicine and Biology, 1997, doi:10.1016/S03015629(97)00143-9.

[3] G.O.N. Oosterhof, E.B. Cornel, G.A.H.J. Smits, F.M.J. Debruyne, J.A. Schalken, "Influence of high-intensity focused ultrasound on the development of metastases," European Urology, 1997, doi: $10.1159 / 000480887$.

[4] N. McDannold, Quantitative MRI-based temperature mapping based on the proton resonant frequency shift: Review of validation studies, International Journal of Hyperthermia, 2005, doi:10.1080/02656730500096073.

[5] K. Hynynen, "Feasibility of using ultrasound phased arrays for MRI monitored noninvasive surgery," IEEE Transactions on Ultrasonics, Ferroelectrics, and Frequency Control, 1996, doi:10.1109/58.542049.

[6] J. Song, A. Pulkkinen, Y. Huang, K. Hynynen, "Investigation of standingwave formation in a human skull for a clinical prototype of a large-aperture, transcranial mr-guided focused ultrasound (MRgFUS) phased array: An experimental and simulation study," IEEE Transactions on Biomedical Engineering, 2012, doi:10.1109/TBME.2011.2174057.

[7] C. Simon, P. Vanbaren, E.S. Ebbini, "Two-dimensional temperature estimation using diagnostic ultrasound," IEEE Transactions on Ultrasonics, Ferroelectrics, and Frequency Control, 1998, doi:10.1109/58.710592.

[8] Y. ISEKI, K. NAKAMURA, D. ANAN, K. KATO, "Development of Ultrasound-Guided Resonant Cavity Applicator System: - Non-invasive Measurement of Temperature Distributions -," Japanese Journal of Hyperthermic Oncology, 2015, doi:10.3191/thermalmed.31.13.

[9] Y. ISEKI, D. ANAN, T. SAITO, Y. SHINDO, F. IKUTA, K. TAKAHASHI, K. KATO, "Non-Invasive Measurement of Temperature Distributions During Hyperthermia Treatments using Ultrasound B-mode Images," Thermal Medicine, 2016, doi:10.3191/thermalmed.32.17.

[10] E. MIURA, K. KATO, A. TAKEUCHI, "Basic Study of 3-D Non-Invasive Measurement of Temperature Distribution Using Ultrasound Images during FUS Heating (article in Japanese with an abstract in English)," Thermal Medicine, 2018, doi:10.3191/thermalmed.34.1.

[11] A.M. Pouch, T.W. Cary, S.M. Schultz, C.M. Sehgal, "In vivo noninvasive temperature measurement by B-mode ultrasound imaging," Journal of Ultrasound in Medicine, 2010, doi:10.7863/jum.2010.29.11.1595.

[12] M.D. Abolhassani, A. Norouzy, A. Takavar, H. Ghanaati, "Noninvasive temperature estimation using sonographic digital images," Journal of Ultrasound in Medicine, 2007, doi:10.7863/jum.2007.26.2.215. 